\title{
Validating a Japanese Version of the Athlete Psychological Strain Questionnaire
}

\author{
Yasutaka Ojio ${ }^{1 *+} \mathbb{0}$, Asami Matsunaga ${ }^{1 \dagger}$, Shin Kawamura ${ }^{2}$, Masanori Horiguchi $^{2}$, Goro Yoshitani ${ }^{2}$, \\ Kensuke Hatakeyama ${ }^{2}$, Rei Amemiya ${ }^{3}$, Ayako Kanie ${ }^{4}$, Rosemary Purcell ${ }^{5,6}$, Simon M. Rice ${ }^{5,6}$ and Chiyo Fujii ${ }^{1}$
}

\begin{abstract}
Background: There is increasing international interest in clinical practice and research related to mental health in the international sports society. The athlete-specific psychological distress assessment tool that addresses potential mental health needs can help promote early detection and recovery of mental illness, as well as physical illnesses. Currently, little is known about the applicability of the useful assessment tool for Japanese elite athletes. The Athlete Psychological Strain Questionnaire (APSQ) is a brief, effective and reliable screening tool to identify early signs of athlete-specific distress and potential mental health symptoms. We examined the applicability and reliability of a Japanese version of the APSQ (APSQ-J) in a Japanese elite athlete context. Further, we examined the construct validity of the APSQ-J.

Methods: We collected web-based anonymous self-report data from 219 currently competing Japanese professional male rugby players. A two-stage process was conducted to validate the factor structure of the APSQ-J using exploratory factor analysis (EFA) in a randomly partitioned calibration sample and confirmatory factor analysis (CFA) in a separate validation sample. Cronbach's alpha is used to assess internal consistency. Pearson product-moment correlation coefficients were calculated to determine if the APSQ-J was significantly associated with measures of psychological distress and well-being using Kessler-6 (K6) and the WHO-5 Well-Being Index, respectively.

Results: We identified a one-factor structure for the APSQ-J. Confirmatory factor analysis supports this one-factor model, revealing good model fit indices. The standardized path coefficients for each of the items were $\beta=0.41-0.83$ $(p<0.001)$. A Cronbach's alpha of 0.84 was obtained for the APSQ-J. The APSQ-J demonstrated significant correlations with the K-6 $(r=0.80, p<0.001)$ and WHO-5 $(r=-0.58, p<0.001)$.

Conclusion: The APSQ-J can be an appropriate and psychometrically robust measure for identifying athlete-specific distress in elite athletes in Japan. Widely disseminating and utilizing this scale in Japanese sports society may support athletes' mental health via early detection of symptoms of psychological distress.
\end{abstract}

Keywords: Mental health, Screening, Rugby player, APSQ, Help-seeking

\section{Key points}

- Athlete Psychological Strain Questionnaire (APSQ) is an athlete-specific psychological distress assessment

*Correspondence: ojio@ncnp.go.jp

†Yasutaka Ojio and Asami Matsunaga have contributed equally to this work.

1 Department of Community Mental Health and Law, National Institute of Mental Health, National Center of Neurology and Psychiatry, 4-1-1 Ogawa-Higashi, Kodaira, Tokyo 187-8553, Japan

Full list of author information is available at the end of the article tool that addresses potential mental health needs.

- Current findings from the sample of Japan Rugby Top League players supported the factor structure, validity, and reliability of the Japanese version of the APSQ (APSQ-J) scale, with a one-factor structure, 
indicating the higher score, the worse the psychological distress and strain.

- APSQ-J is a brief and effective evaluation tool that facilitates the development and social implementation of mental health support for elite athletes in Japan.

\section{Background}

Recent research and clinical practice for mental health in elite athletes has increased, similar to the trajectory for physical health and injury care. Most of the findings have come from the USA [1, 2], European countries [3-6], and Australasia [7, 8], with studies from Asia still limited [9, 10]. These findings consistently demonstrate that mental health symptoms are common in elite athletes [11]. Contributing factors include athlete-specific factors (e.g. physical injury, poor performance, competition for selection, career transition) and general factors, such as stressful life events and inadequate social support $[6,8,9,11$, $12]$. Several statements presented by international sports organizations and experts call for mental health care systems and environmental improvements for athletes [11, 13-20]. Mental health status affects competitiveness, as well as non-competitive daily living, and delays in support and care for poor mental health can worsen symptoms [21]. An athlete-specific mental health assessment tool can help facilitate early detection and recovery from mental ill-health, as with physical illness.

The Athlete Psychological Strain Questionnaire (APSQ) is a mental health assessment tool that addresses these potential needs specifically within an elite sports context [22, 23]. The APSQ is a 10-item scale developed by Rice and colleagues using survey data on Australian male athletes [22] and also validated with female elite athletes [23]. The authors created the item pool from mental health research findings related to athletes, and the preliminary scale, validated in male athletes, consisted of 10 items loading onto three factors, namely Self-Regulation, Performance, and External Coping.

The APSQ is included as the triage measure in the Sports Mental Health Assessment Tool-1 (SMHAT-1), a mental health screening procedure developed by the International Olympic Committee (IOC) to better support the mental health of athletes and stimulate more international research [24]. At present, little is known about the applicability of the APSQ to Japanese elite athletes. The development of a Japanese version (the APSQJ) may promote mental health research and practice for elite athletes in Asia, including Japan. Therefore, this study aims to develop and validate a Japanese version of the APSQ in a sample of Japanese male professional rugby players. In addition, the other aim is to compare the means of APSQ-J with the original APSQ from the sample of Australian male elite athletes to understand how similar they are.

\section{Methods \\ Study Design and Setting}

The current study employed a web-based cross-sectional design. We followed the Strengthening the Reporting of Observational Studies in Epidemiology (STROBE) guidelines for observational cross-sectional studies (Additional file 1) [25]. We distributed the survey weblink (URL) to all players in the Japan Rugby Players' Association through one or two team representatives participating in regular meetings. The potential participants were invited to complete the anonymous survey. They were informed about the survey process, including the purpose of the study, data collection procedures, and the consequences of participating or not participating via the cover page of the questionnaire. Consenting participants went on to complete the questionnaire, which took less than $10 \mathrm{~min}$. The participants were provided with individual one-time access to the survey using IP address filtering access to a tablet or laptop computer to complete the survey. The cross-sectional data were collected shortly before the start of the off-season from December 2020 to February 2021.

\section{Participants}

We collected data from a total of 612 rugby players (565 Japanese players and 47 foreign players) registered with the Japan Rugby Players Association. The participants were all aged 18 years and over and belonged to the Japan Rugby Top League. The current survey was available in both Japanese and English. No exclusion criteria were applied. Overall, 227 of the 612 players agreed to complete the survey (response rate: $37.1 \%$ ). The response rate of this survey was consistent with other mental health surveys in Japan [26]. We analysed 219 out of 227 participants born in Japan as the target population in the current study. All investigators received the learning course on research ethics, and this study was approved and facilitated by the Research Ethics Committee at the National Center of Neurology and Psychiatry (approval number: A2020-058).

\section{Measurements}

\section{The Athlete Psychological Strain Questionnaire (APSQ)}

The APSQ is a brief, self-report questionnaire specific to the elite sporting context, comprised of 10 items (e.g. 'During the past 4 weeks, I could not stop worrying about injury or my performance') scored on a 5-point scale (from 'none of the time' (1) to 'all of the time' (5)). A total score ranging from 10 to 50 , with higher scores 
representing more psychological distress, is calculated by summing the answers on the 10 items [22]. A more recent study has shown that scores of $\geq 15, \geq 17$ and $\geq 20$ represent moderate, high and very high levels of athlete-specific distress [23]. In the preliminary stage of developing the original version of APSQ [22], a two-step approach was used in a sample of 1007 currently competitive Australian elite male athletes from professional sports $(M=23.67, \mathrm{SD}=4.16)$. The exploratory and confirmatory factor analysis and tests of differential item functioning were conducted with the samples randomly partitioned into calibration $(n=497)$ and validation $(n=510)$ samples. Exploratory factor analysis, with parallel analysis, conducted on the calibration sample supported a secondorder with three-factor model. The subscales included Self-Regulation, Performance, and External Coping domains, accounting for $50.44 \%$ of total scale variance. In the second-order model, the path coefficient from the upper factor "Athlete psychological strain" to each factor was 0.8 or more. In the confirmatory factor analysis, excellent model goodness-of-fit indicators were provided. The mean score for the 10 items among the Australian male athletes was $14.67(\mathrm{SD}=5.47)$.

For this study, the original APSQ was translated into Japanese by the first author (Y.O.). The professional athletes (S.K. and K.H.) in this research team modified the terms and sentences to improve the readability for athletes. A bilingual English speaker then produced a backtranslation. Finally, the back-translated version of the APSQ was confirmed and approved by the researchers who had originally developed the APSQ (S.M.R and R.P).

\section{Other Measurements}

We used the Kessler-6 (K6) and the WHO-5 Well-Being Index, widely used measures of psychological distress and well-being to assess convergent validity with the APSQ-J. Previous studies have shown that the performance of the K6 in screening mood and anxiety disorders, as assessed by the areas under the receiver operating characteristic curves (AUCs), was excellent [27], with values as high as $0.86,0.89$, and 0.94 from US [28], Australian [29], and Japanese general samples [30], respectively. In the K-6, the scores are categorized to indicate the respondents' mental health status over the previous 30 days. Responses to items are made on a 5-point scale. The K-6 was developed and validated based on many epidemiological surveys and is widely used as a screening tool in assessing treatment progress in common mental disorders such as anxiety and depression in people in the general community [31]. Cronbach's $\alpha$ was 0.91 in the present sample for the K6. The WHO-5 Well-Being Index is a 5-item self-report scale that assesses positive aspects of mental health (i.e. "I have felt cheerful and in good spirits") over the previous 2 weeks $[32,33]$. The raw score is calculated by totalling the figures of a 5 -point scale (i.e. $0=$ "at no time"; $5=$ "All of the time"). The score ranges from 0 to 25 , with 0 representing the worst possible and 25 representing the best possible well-being. A score below 13 indicates poor well-being and is an indication for assessing for depression according to the ICD-10. Cronbach's $\alpha$ was 0.90 in the present sample for the WHO-5.

\section{Background Information/Demographics}

The background information and demographic survey items included age, country of birth, educational attainments, marital status, the number (if any) of dependent children, residential status, the national team's experience, and playing status for the last season.

\section{Statistical Methods}

Descriptive statistics were used to characterize the sample. We conducted a two-stage process to validate the factor structure of the APSQ-J using exploratory factor analysis (EFA) in a randomly partitioned calibration sample and confirmatory factor analysis (CFA) in a separate validation sample. EFA was undertaken to determine the underlying factor structure of the APSQ-J. To determine which items belonged to each factor, we extracted items if they loaded $\geq 0.3$. In addition, we examined the number of factors based on scree plots. We adopted the oblique rotation (promax) with principal factor method. In CFA, we evaluated the fit of the model with the data using the chi-squared statistic (CMIN), root mean square error of approximation (RMSEA), and comparative fit index (CFI). According to conventional criteria, an excellent fit would be indicated by $\mathrm{CMIN} / \mathrm{df}<2$, RMSEA $<0.05$, CFI $>0.97$, while $\mathrm{CMIN} / \mathrm{df}<3$, RMSEA $<0.08$, CFI $>0.95$ demonstrates an acceptable fit [34]. After confirming the factor structure, we examined the internal consistency and convergent and divergent validity. To evaluate internal consistency, the Item-test, Item-rest correlation and Cronbach's alpha was calculated. In terms of convergent validity, Pearson product-moment correlation coefficients were calculated to determine if the APSQ-J correlated significantly with the K-6 and WHO-5. Therefore, we determined convergent and divergent validity through examining patterns of statistically significant correlations with the K-6 (convergent validity evidenced by significant positive associations) and the WHO-5 (divergent validity evidenced by significant negative associations). We conducted a one-sample $t$ test for comparing the current data to the means reported in developing the APSQ paper from the 1007 currently competing Australian elite male athletes [22]. For the effect size of the mean 
Table 1 Demographic characteristics of the study participants

$\%(n)$

Age at survey

$\begin{array}{lc}20-24 & 27.85(61) \\ 25-29 & 45.66(100) \\ 30-34 & 21.46(47) \\ 35- & 5.02(11) \\ \text { Educational attainment } & \\ \text { High school } & 1.82(4) \\ \text { Four-year college or university } & 97.3(213) \\ \text { Postgraduate college (or higher) } & 0.91(2) \\ \text { Marital status } & \\ \text { Married } & 47.49(104) \\ \text { Never married } & 51.60(113) \\ \text { Divorced or widowed } & 0.91(2) \\ \text { Child living in household } & \\ \text { No } & 75.34(165) \\ \text { Yes } & 24.66(54) \\ \text { Residential status } & \\ \text { Living alone } & 21.92(48) \\ \text { Living with family and/or partner } & 47.95(105) \\ \text { Dormitory } & 30.14(66) \\ \text { Experience of national team } & \\ \text { No } & 80.37(176) \\ \text { Yes } & 19.63(43) \\ \text { Playing status of last season } & \\ \text { As an active member } & 34.25(75) \\ \text { As a reserve member } & 28.77(63) \\ \text { No play } & 36.99(81)\end{array}$

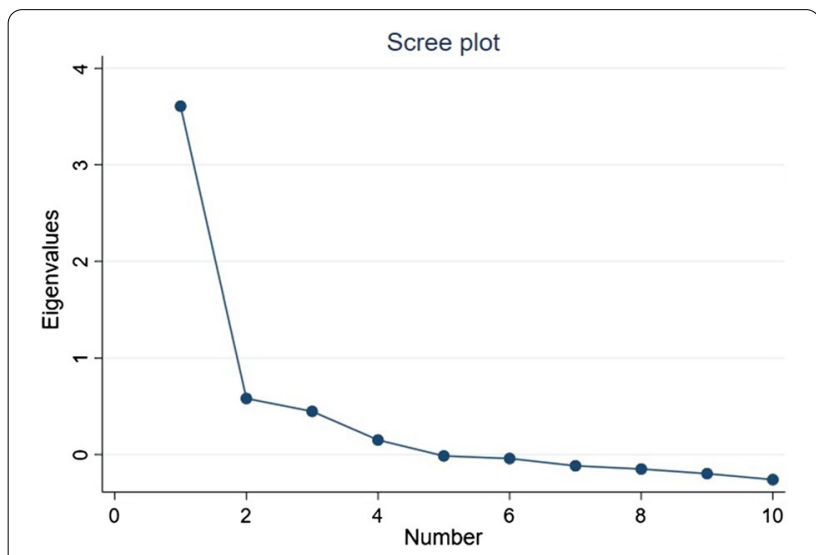

Fig. 1 Scree plots and parallel analysis for APSQ-J

difference between the two countries in each assessment point, through computing Cohen's d, which was graded as $0.20=$ small, $0.50=$ medium, $0.80=$ large. All analyses were conducted with Stata version 16 (StataCorp LLC, College Station, TX, US).

\section{Results}

We analysed 219 players born in Japan. The demographic characteristics of the participants are shown in Table 1. Almost half were 25-29 years old; the overwhelming majority had graduate university educational attainment and one in five players reported experience in the national team.

In EFA, considering the results of the scree plots (Fig. 1), we employed a one-factor structure as optimal. Factor loadings of the items are shown in Table 2. When the one-factor structure was employed, the factor loading values for all the items were $\geq 0.3$.

The results of CFA are presented in Fig. 2. The onefactor model of APSQ-J showed the following model fit indices: $\quad \mathrm{CMIN} / d f=2.48 \quad(p<0.001), \quad \mathrm{RMSEA}=0.117$, $\mathrm{CFI}=0.871$. Along with modification indices which suggest a high parameter change, covariances were added between some of the error variables of items. After the modification, we obtained a final model with excellent fit indices: $\mathrm{CMIN} / d f=1.024(p<0.001), \mathrm{RMSEA}=0.015$; $\mathrm{CFI}=0.998$. The standardized path coefficients for each item were $\beta=0.41-0.83(p<0.001)$.

The Item-test, Item-rest correlation for the 10 -items APSQ-J is shown in Table 3. The Cronbach's alpha $(\alpha)$ of 0.84 was obtained for the APSQ-J. APSQ-J showed a significant strong positive correlation with K-6 $(r=0.80$, $p<0.001)$ and a significant moderate negative correlation with WHO-5 $(r=-0.58, p<0.001)$. The total score for the 10 items was significantly higher than that for Australians $($ mean $=19.16(\mathrm{SD}=6.30)$ vs. mean $=14.67$ $(\mathrm{SD}=5.47), \mathrm{t}=10.70 ; \mathrm{df}=1224 ; p<0.001)$ in Additional file 1: Table S1. The effect size for the difference was almost large (Cohen's d: 0.76).

\section{Discussion}

This study evaluated the psychometric properties of the Japanese version of APSQ in order to contribute to improving mental health promotion and support for Japanese elite athletes. We confirm the factor structure, validity, and reliability of the scale in Japanese male professional rugby players. The APSQ-J demonstrated a onefactor structure which is different from the three-factor of the original version of APSQ from the factor analysis. The APSQ-J could be considered a useful assessment tool for athlete-specific psychological distress in Japanese elite athletes, with relatively high Cronbach's alphas, as well as convergent validity with the K-6 and WHO-5.

The Japanese version of APSQ did not match the factor structure of the original version of APSQ. The original 
Table 2 Factor loadings for APSQ-J items

\begin{tabular}{lll}
\hline Item no & Statement & Factor loadings \\
\hline 1 & It was difficult to be around teammates & 0.60 \\
2 & I found it difficult to do what I needed to do & 0.75 \\
3 & I was less motivated & 0.76 \\
4 & I was irritable, angry or aggressive & 0.58 \\
5 & I could not stop worrying about injury or my performance & 0.63 \\
6 & I found training more stressful & 0.74 \\
7 & I found it hard to cope with selection pressures & 0.61 \\
8 & I worried about life after sport & 0.49 \\
9 & I needed alcohol or other substances to relax & 0.31 \\
10 & It was difficult to be around teammates & 0.37 \\
\hline
\end{tabular}
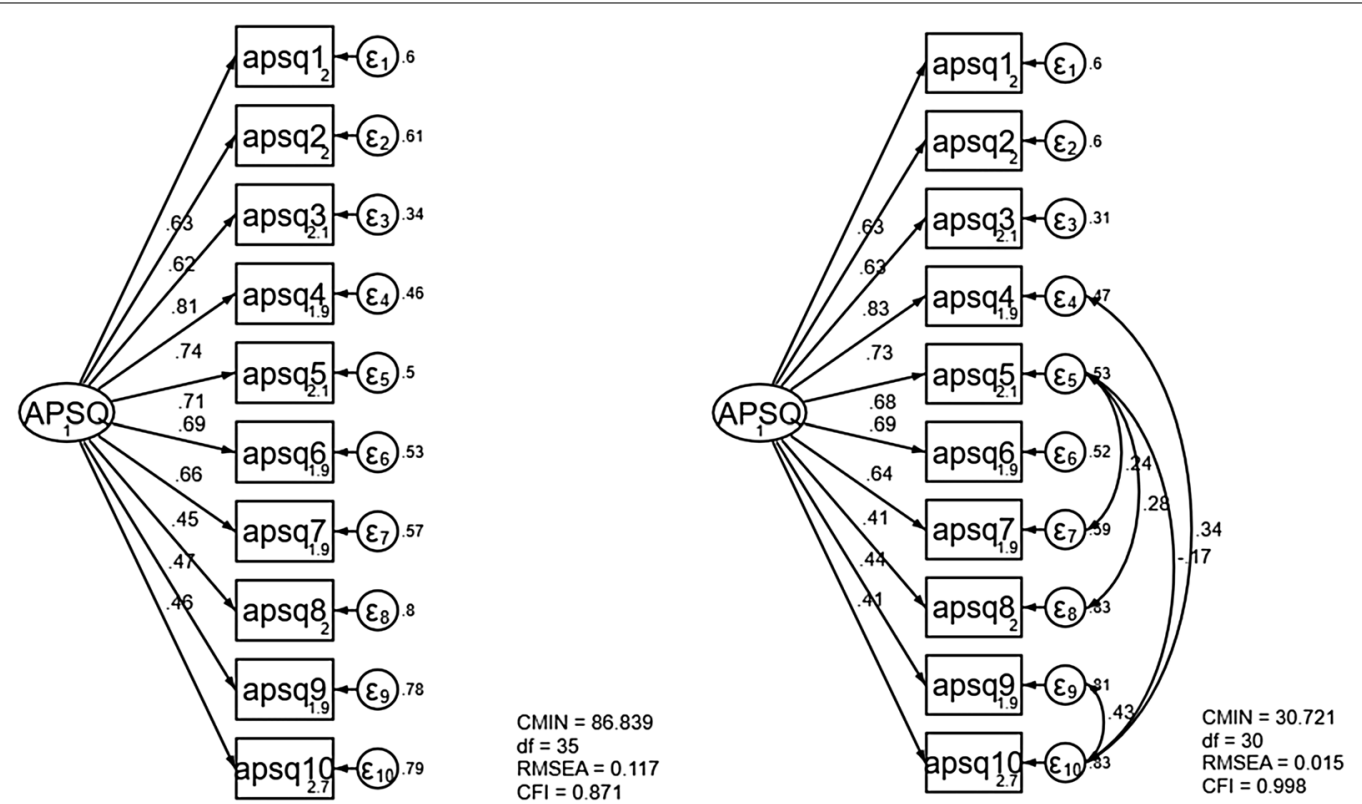

Fig. 2 Standardized regression weights for the APSQ-J one-factor structure. Abbreviation: Chi-square statistics (CMIN); root mean square error of approximation (RMSEA); comparative fit index (CFI); Tacker-Lewis fit index (TLI)

version had three factors: Self-regulation, Performance, and External Coping [22, 23], while the Japanese version had one factor. The differences might reflect behavioural or reaction patterns related to psychological distress and mental health that are closely associated with national cultures. For example, Japanese culture has a "tightness" social norm and low tolerance of deviant behaviour to enhance order and social coordination to effectively deal with threats to social cohesion [35]. Emphasizing such harmonious social relationships tends to prefer self-improvement rather than externalization as a coping action [36]. In addition, people in Japan also tend to hesitate to seek help due to a strong influence of stigma compared to Western countries [37, 38]. In addition, this study was a preliminary survey involving only male rugby players which may have further influenced the results. In the Japanese sports society, where there is little research on the mental health of elite athletes, the development of this preliminary scale provides an opportunity for more research in the future.

Our findings suggest that the APSQ-J confirmed a good convergent and divergent validity with the K-6 and WHO-5, respectively. The advantage of using the APSQ-J in elite athlete populations is the ability to detect athletespecific distress, which can then enable mental health support for affected athletes. The APSQ is a 10-item, 
Table 3 Item-test, item-rest correlation and Cronbach's alpha of the APSQ-J

\begin{tabular}{llll}
\hline Item no & $\begin{array}{l}\text { Item-test } \\
\text { correlation }\end{array}$ & $\begin{array}{l}\text { Item-rest } \\
\text { correlation }\end{array}$ & $\boldsymbol{a}$ \\
\hline 1 & 0.63 & 0.54 & 0.83 \\
2 & 0.70 & 0.61 & 0.82 \\
3 & 0.78 & 0.70 & 0.81 \\
4 & 0.68 & 0.59 & 0.83 \\
5 & 0.75 & 0.64 & 0.82 \\
6 & 0.74 & 0.65 & 0.82 \\
7 & 0.71 & 0.59 & 0.83 \\
8 & 0.59 & 0.44 & 0.84 \\
9 & 0.45 & 0.36 & 0.84 \\
10 & 0.44 & 0.39 & 0.84 \\
Test scale & & & 0.84 \\
\hline
\end{tabular}

sufficiently brief tool highly relevant to the competitive sport context, by its inclusion of items related to injury, training, and selection, as well as mental health-related externalization issues such as alcohol and substance abuse. While $\mathrm{K} 6$ focuses on internalized symptoms, the APSQ includes several externalized symptoms in addition to such internalized symptoms. This feature was included as elite athletes conforming to traditional masculinity norms may be more likely to experience and manifest externalized, rather than internalized symptoms, as a sign of poor mental health [11, 39]. Focusing on the response to each item may also help provide practical mental health support for athletes.

The current study showed that the items of APSQ-J are summed as factor to generate a total score, suggesting that the higher the score, the worse the psychological distress and strain. We also demonstrated that the total score in the sample of Japanese male rugby players was higher than that of Australian male elite athletes. Considering the difference of the total score in each country, the next step in developing the APSQ-J will be to further explore the validity of APSQ-J cut-off values, including relative to the original version [23]. Furthermore, the APSQ-J can also be utilized in the Japanese version of SMHAT-1 that is under development by the authors [24].

\section{Strengths and Limitations of this Research}

The major strengths of this study were developing a Japanese version of the APSQ and confirming its psychometric properties. Our efforts are expected to advance mental health research among elite athletes in Japan, which has been delayed in comparison with the USA, European countries and Australia. In translating the scale development process, we coordinated athlete-friendly terminology with active elite athletes. This co-production with elite athletes may have improved the accuracy of answering APSQ-J questions. However, we also recognize several limitations that may impact the findings. The current samples were all male professional rugby players and the response rate was less than $40 \%$, although this is consistent with other mental health surveys in Japan. Future research should aim to examine the psychometric properties of the APSQ-J in a wider, more representative cohort, including female athletes and those from other sports. Furthermore, because we employed a cross-sectional survey design, the test-retest for the stability of the APSQ-J was not evaluated. Finally, the effectiveness of scale convergence and divergence could be better understood by consideration of critical stages of risk for athlete mental health, such as injury duration and severity and the transition from competitive sports.

\section{Conclusions}

We have developed the APSQ-J, which enables screening for mental health status in Japanese elite athletes. Utilizing this scale may support athletes' mental health via early detection of symptoms of psychological distress. There is increasing international interest in clinical practice and research related to mental health in the international sports society. APSQ-J, a brief and effective evaluation tool, is expected to be widely used in the Japanese sports society. In addition, early detection of psychological distress by this tool may help promote mental health support for elite athletes.

\section{Abbreviations \\ APSQ: Athlete Psychological Strain Questionnaire; APSQ-J: Japanese version of the Athlete Psychological Strain Questionnaire; EFA: Exploratory factor analysis; CFA: Confirmatory factor analysis; K6: Kessler-6; SMHAT-1: Sports Mental Health Assessment Tool-1; IOC: International Olympic Committee; STROBE: Strength- ening the Reporting of Observational Studies in Epidemiology; JRPA: Japan Rugby Players' Association; SD: Standard Deviation; RMSEA: Root mean square error of approximation.}

\section{Supplementary Information}

The online version contains supplementary material available at https://doi. org/10.1186/s40798-021-00385-9.

Additional file 1. Comparison of Means and SDs of participants' responses to APSQ between Japanese and Australian elite athletes.

\section{Acknowledgements}

Ms Miyako Fukuda and Dr Fumie Arie cooperated with us on the ethical considerations in the implementation of this study. We also received generous assistance from Mr Masato Kotake, MSc. We would also like to thank Handfast Point for English editing support with the paper.

\section{Authors' Contributions}

Y.O, A.M, S.M.R and R.P and C.F contributed to writing the draft manuscript and the statistical analysis. Y.O, A.M, M.H, R.A, A.K, and C.F, contributed to the conception and design of the study. S.K, M.H, G.Y, and K.H contributed to this 
survey planning, data collection and management. All the authors contributed to the data assessment and approved the final version of the manuscript.

\section{Funding}

This study was supported by grants from the TOYOTA foundation. The funders had no role in study design, data collection and analysis, decision to publish, or manuscript preparation. The foundation did not provide support for the salaries of all authors. Japan Rugby Players' Association (JRPA) is not a commercial company and does not support salaries for any authors. In addition, JRPA did not provide financial support for this study and any other related research processes.

\section{Availability of Data and Materials}

Data cannot be shared publicly because there exist ethical restrictions. Publishing data sets is not covered by the informed consent provided by the study participants, which was approved by the ethics committee of the Research Ethics Committee at the National Center of Neurology and Psychiatry. The data are not owned by a third party. Non-author contact information for the body imposing the restrictions upon the data, to which data access requests can be sent, is the Research Ethics Committee of the National Center of Neurology and Psychiatry (rinri-jimu@ncnp.go.jp).

\section{Declarations}

\section{Ethics Approval and Consent to Participate}

This study was performed in accordance with the standards of ethics outlined in the Declaration of Helsinki and approved by the Research Ethics Committee at the National Center of Neurology and Psychiatry, Japan (NCNP) (approval number: A2020-058)

\section{Consent for Publication}

The participants were informed about the survey process, including the purpose of the study, data collection procedures, and the consequences of participating or not participating via the cover page of the questionnaire. Each participant gave their informed consent to use their response for research purposes.

\section{Competing interests}

The authors declare that they have no competing interests.

\section{Author details}

${ }^{1}$ Department of Community Mental Health and Law, National Institute of Mental Health, National Center of Neurology and Psychiatry, 4-1-1 Ogawa-Higashi, Kodaira, Tokyo 187-8553, Japan. ${ }^{2}$ Japan Rugby Players' Association, Tokyo, Japan. ${ }^{3}$ Faculty of Health and Sports Sciences, University of Tsukuba, Tsukuba, Ibaraki, Japan. ${ }^{4}$ National Center for Cognitive Behavioral Therapy and Research, National Center of Neurology and Psychiatry, Tokyo, Japan. ${ }^{5}$ Orygen, Parkville, VIC, Australia. ${ }^{6}$ Centre for Youth Mental Health, The University of Melbourne, Melbourne, VIC, Australia.

Received: 27 June 2021 Accepted: 18 November 2021 Published online: 11 December 2021

\section{References}

1. Rao AL, Asif IM, Drezner J, Toresdahl BG, Harmon KG. Suicide in National Collegiate Athletic Association (NCAA) athletes: a 9-year analysis of the NCAA resolutions database. Sports Health. 2014;7:452-7.

2. Wolanin A, Hong E, Marks D, Panchoo K, Gross M. Prevalence of clinically elevated depressive symptoms in college athletes and differences by gender and sport. Br J Sports Med. 2016;50:167-71.

3. Gouttebarge V, Backx FJ, Aoki H, Kerkhoffs GM. Symptoms of common mental disorders in professional football (soccer) across five European countries. J Sports Sci Med. 2015;14:811-8.

4. Nicholls AR, Madigan DJ, Fairs LRW, Bailey R. Mental health and psychological well-being among professional rugby league players from the UK. BMJ Open Sport Exerc Med. 2020;6:e000711.
5. Junge A, Feddermann-Demont N. Prevalence of depression and anxiety in top-level male and female football players. BMJ Open Sport Exerc Med. 2016;2(1):e000087.

6. Nixdorf I, Frank R, Hautzinger M, Beckmann J. Prevalence of depressive symptoms and correlating variables among German elite athletes. J Clin Sport Psychol. 2013;7:313-26.

7. Gulliver A, Griffiths KM, Mackinnon A, Batterham PJ, Stanimirovic R. The mental health of Australian elite athletes. J Sci Med Sport. 2015;28:255-61.

8. Purcell R, Rice S, Butterworth M, Clements M. Rates and correlates of mental health symptoms in currently competing elite athletes from the Australian national high-performance sports system. Sports Med. 2020;50(9):1683-94.

9. Ojio Y, Matsunaga A, Hatakeyama K, Kawamura S, Horiguchi M, Yoshitani $\mathrm{G}$, et al. Anxiety and depression symptoms and suicidal ideation in Japan rugby top league players. Int J Environ Res Public Health. 2021;18(3):1205.

10. Chen X, Qiu N, Chen C, Wang D, Zhang G, Zhai L. Self-efficacy and depression in boxers: a mediation model. Front Psychiatry. 2020;11:00791.

11. Reardon CL, Hainline B, Miller AA, Baron D, Baum AL, Bindra A, et al. Mental health in elite athletes: International Olympic Committee consensus statement (2019). Br J Sports Med. 2019;53:667-99.

12. Rice SM, Gwyther K, Santestebanecha O, Baron D, Gorczynski P, Gouttebarge $V$, et al. Determinants of anxiety in elite athletes: a systematic review and meta-analysis. Br J Sports Med. 2019;53:722-30.

13. Breslin G, Smith A, Donohue B, Donnelly P, Shannon S, Haughey TJ, et al. International consensus statement on the psychosocial and policy related approaches to mental health awareness programmes in sport. BMJ Open Sport Exerc Med. 2019;5:e000585.

14. Chang C, Putukian M, Aerni G, Diamond AB, Hong ES, Ingram YM, et al. Mental health issues and psychological factors in athletes: Detection, management, effect on performance and prevention: American medical society for sports medicine position statement-executive summary. Br J Sports Med. 2020;54:216-20.

15. Gorczynski P, Gibson K, Thelwell R, et al. The BASES expert statement on mental health literacy in elite sport. Sport Exercise Sci. 2019;59:6-7.

16. Henriksen K, Schinke R, McCann S, et al. Athlete mental health in the olympic/paralympic quadrennium: a multi-societal consensus statement. Int J Sport Exerc Psychol. 2020;2020(18):391-408.

17. Henriksen K, Schinke R, Moesch K, Anthony P, Chris H, Florence K. Consensus statement on improving the mental health of high performance athletes. Int J Sport Exerc Psychol. 2019;18(5):553-60.

18. Moesch K, Kentta G, Kleinert J, Quignon-Fleuret C, Cecil S, Bertollo M. FEPSAC position statement: mental health disorders in elite athletes and models of service provision. Psychol Sport Exerc. 2019;38:61-71.

19. Schinke RJ, Stambulova NB, Si G, Moore Z. International society of sport psychology position stand: Athletes' mental health, performance, and development. Int J Sport Exerc Psychol. 2018;16:622-39.

20. Van Slingerland KJ, Durand-Bush N, Lindsay B, Goldfield G, Archambault R, Smith D, et al. Canadian Centre for mental health and sport (CCMHS) position statement: principles of mental health in competitive and high performance sport. Clin J Sport Med. 2019;29:173-80.

21. Purcell R, Gwyther K, Rice SM. Mental health in elite athletes: increased awareness requires an early intervention framework to respond to athlete needs. Sports Med - Open. 2019;5:46.

22. Rice SM, Parker AG, Mawren D, Clifton P, Harcourt P, Lloyd M, et al. Preliminary psychometric validation of a brief screening tool for athlete mental health among male elite athletes: the athlete psychological strain questionnaire. Int J Sport Exerc Psychol. 2019;18(6):850-65.

23. Rice S, Olive L, Gouttebarge V, Clifton P, Parker AG, Clifton P, Harcourt P, et al. Mental health screening: severity and cut-off point sensitivity of the athlete psychological strain questionnaire in male and female elite athletes. BMJ Open Sport Exerc Med. 2020;6:e000712.

24. Gouttebarge V, Bindra A, Blauwet C, Campriani N, Currie A, Engebretsen L, et al. International Olympic Committee (IOC) Sport Mental Health Assessment Tool 1 (SMHAT-1) and Sport Mental Health Recognition Tool 1 (SMHRT-1): towards better support of athletes' mental health. Br J Sports Med. 2021;55(1):30-7.

25. von Elm E, Altman DG, Egger M, Pocock SJ, Gøtzsche PC, Vandenbroucke JP. STROBE initiative. The strengthening the reporting of observational studies in epidemiology (STROBE) statement: guidelines for reporting observational studies. J Clin Epidemiol. 2008;61:344-9. 
26. Kawakami N, Yasuma N, Watanabe K, Ishikawa H, Tachimori H, Takeshima T, et al. Association of response rate and prevalence estimates of common mental disorders across 129 areas in a nationally representative survey of adults in Japan. Soc Psychiatry Psychiatr Epidemiol. 2020;55(10):1373-82.

27. Prochaska JJ, Sung HY, Max W, Shi Y, Ong M. Validity study of the K6 scale as a measure of moderate mental distress based on mental health treatment need and utilization. Int J Methods Psychiatr Res. 2012;21:88-97.

28. Kessler RC, Barker PR, Colpe LJ, Epstein JF, Gfroerer JC, Hiripi E, et al. Screening for serious mental illness in the general population. Arch Gen Psychiatry. 2003;60:184-9.

29. Furukawa TA, Kessler RC, Slade T, Andrews G. The performance of the K6 and K10 screening scales for psychological distress in the Australian national survey of mental health and well-being. Psychol Med. 2003;33:357-62.

30. Furukawa TA, Kawakami N, Saitoh M, Ono Y, Nakane Y, Nakamura Y, et al. The performance of the Japanese version of the K6 and K10 in the world mental health survey Japan. Int J Methods Psychiatr Res. 2008;17:152-8.

31. Nishi D, Imamura K, Watanabe K, Ishikawa H, Tachimori H, Takeshima T, et al. Psychological distress with and without a history of depression: results from the World Mental Health Japan 2nd Survey (WMHJ2). J Affect Disord. 2020;265:545-51.

32. Awata S, Bech P, Koizumi Y, Seki T, Kuriyama S, Hozawa A, et al. Validity and utility of the Japanese version of the WHO-Five Well-Being Index in the context of detecting suicidal ideation in elderly community residents. Int Psychogeriatr. 2007;19:77-88.

33. Topp CW, Østergaard SD, Søndergaard S, Bech P. The WHO-5 Well-Being Index: a systematic review of the literature. Psychother Psychosom. 2015;84(3):167-76.

34. Schermelleh-Engel K, Moosbrugger H, Müller H. Evaluating the Fit of structural equation models: tests of significance and descriptive goodness-of-fit measures. Methods Psychol Res. 2003;8(8):23-74.

35. Gelfand MJ, Raver JL, Nishii L, Leslie LM, Lun J, Lim BC, et al. Differences between tight and loose cultures: a 33-nation study. Science. 2011;332(6033):1100-4.

36. Uchida Y, Kitayama S. Happiness and unhappiness in east and west: themes and variations. Emotion. 2009;9(4):441-56.

37. Ting JY, Hwang WC. Cultural influences on help-seeking attitudes in Asian American students. Am J Orthopsychiatry. 2009;79:125-32.

38. Mojaverian T, Hashimoto T, Kim HS. Cultural differences in professional help seeking: a comparison of Japan and the U.S. Front Psychol. 2013;3:615.

39. Oliffe JL, Rossnagel E, Seidler ZE, Kealy D. Men's depression and suicide. Curr Psychiatry Rep. 2019;21:103.

\section{Publisher's Note}

Springer Nature remains neutral with regard to jurisdictional claims in published maps and institutional affiliations.

\section{Submit your manuscript to a SpringerOpen ${ }^{\circ}$ journal and benefit from:}

- Convenient online submission

- Rigorous peer review

- Open access: articles freely available online

- High visibility within the field

- Retaining the copyright to your article

Submit your next manuscript at $\boldsymbol{\text { springeropen.com }}$ 